\title{
A note on the comparison of mixed-effects models for longitudinal studies
}

\author{
Alicja Szabelska-Beręsewicz', Agnieszka Bilska², Katarzyna Waszkowiak², \\ Idzi Siatkowski ${ }^{1}$

\footnotetext{
${ }^{1}$ Department of Mathematical and Statistical Methods, Poznan University of Life Sciences, Wojska Polskiego 28,60-637 Poznań, Poland, e-mail: aszab@up.poznan.pl, idzi@up.poznan.pl ${ }^{2}$ Institute of Meat Technology, Poznan University of Life Sciences, Wojska Polskiego 31, 60-624 Poznań, Poland, e-mail: abilska@up.poznan.pl,kwaszkowiak@up.poznan.pl
}

\section{SUMMARY}

\begin{abstract}
This paper concerns methods of choosing appropriate models for longitudinal studies. Attention is paid to three criteria: the marginal Akaike Information Criterion (mAIC), the conditional Akaike Information Criterion (cAIC), and the corrected conditional Akaike Information Criterion (ccAIC). We consider these criteria based on an example concerning the effect of storage time and addition of flaxseed (Linum usitatissimum L.) preparations (i.e. ground flaxseeds, defatted flaxseed meal and flaxseed ethanolic extract) on changes in lipid oxidation and fatty acid composition during the storage of liver pâté with partial substitution of fat with flax oil.
\end{abstract}

Key words and phrases: fixed and mixed models, marginal Akaike Information Criterion, conditional Akaike Information Criterion, corrected conditional Akaike Information Criterion

\section{Introduction}

The characteristic of longitudinal studies is that the response variable of interest and a set of explanatory variables are measured several times on each individual in the study. The main objective in such a study is to characterize change in the repeated values of the response variable and to determine the explanatory variables most associated with any change. Since some of the observations of the response variable are made on the same individual, it is likely that the measurements will be correlated rather than independent, even after conditioning on the explanatory variables. Consequently, repeated measures 
data require special methods of analysis, and models for such data need to include parameters linking the explanatory variables to the repeated measurements. One of the possible solutions to this problem is to use a mixedeffect model, where one of the random effects included in the model will be connected to the individual. For such a model, we would like to determine which explanatory variables are significant. For this purpose it is possible to use the marginal Akaike Information Criterion (mAIC) (Akaike, 1974). However, this coefficient was initially introduced for a fixed model, which means that it does not include the structure of the mixed-effect model. We shall therefore include in our analysis two additional criteria: the conditional Akaike Information Criterion (cAIC) (Vaida and Blanchard, 2005), and the corrected conditional Akaike Information Criterion (ccAIC) (Liang et al., 2008; Greven and Kneib, 2010).

In section 2 we introduce the considered model and describe the methods of comparison that we will use. That section also presents the material on the effect of storage time and addition of flaxseed (Linum usitatissimum L.) preparations (i.e. ground flaxseeds, defatted flaxseed meal and flaxseed ethanolic extract) on changes in lipid oxidation and fatty acid composition during storage of liver pâté with partial substitution of fat with flax oil, to be used as an example of the methodology presented in the paper. In section 3 we present the results of the estimation of parameters and comparison of the proposed models. In section 4 we discuss the results of the presented example as well as conclusions concerning the proposed methods of comparison.

\section{Material and Methods}

\subsection{Models and methods of their comparison}

We are interested in datasets where samples are collected repeatedly at several time points. For such longitudinal data we consider the following model:

$$
\mathbf{Y}=\mathbf{X} \boldsymbol{\beta}+\mathbf{Z} \mathbf{u}+\boldsymbol{\varepsilon},
$$


where $\mathbf{Y}$ is the $\mathrm{n} \times 1$ vector of observed values, $\mathbf{X}$ and $\mathbf{Z}$ are the known design matrices $(n \times p$ and $n \times r)$ corresponding to the fixed and random effects respectively, $\boldsymbol{\beta}$ is the $\mathrm{p} \times 1$ vector of unknown fixed parameters, $\mathbf{u}$ is the $r \times 1$ vector of random effects, and $\boldsymbol{\varepsilon}$ is the $\mathrm{n} \times 1$ vector of unobservable random error, $\mathbf{u} \sim \mathrm{N}(\mathbf{0}, \mathbf{D}), \boldsymbol{\varepsilon} \sim \mathrm{N}\left(\mathbf{0}, \sigma^{2} \mathbf{I}\right), \mathbf{u}$ and $\boldsymbol{\varepsilon}$ independent. For this general version of the model we would like to determine what specific effects should be included in the model.

To be able to compare the two models we need to introduce appropriate methods of comparison. For fixed models there are several possible methods that evaluate the effectiveness of a model. One commonly used method is based on the marginal perspective on the mixed model

$$
\mathbf{Y} \sim \mathrm{N}(\mathbf{X \beta}, \mathbf{V}),
$$

where $\mathbf{V}=\operatorname{Cov}(\mathbf{Y})=\mathbf{Z D Z} \mathbf{Z}^{\prime}+\sigma^{2} \mathbf{I}$.

Then, the unknown parameters are $(\beta, \theta)$, where $\theta$ contains the unknown parameters in $\mathbf{D}$ and $\mathbf{V}$. Using this perspective we can introduce the loglikelihood for $\beta$ and $\theta$ as:

$$
\ell(\beta, \theta)=-\frac{\mathrm{n}}{2} \log (2 \pi)-\frac{1}{2} \log |\mathbf{V}|-\frac{1}{2}(\mathbf{Y}-\mathbf{X} \boldsymbol{\beta})^{\prime} \mathbf{V}^{-1}(\mathbf{Y}-\mathbf{X} \boldsymbol{\beta}) .
$$

The selection of the model can be based on the mAIC, which has the following form:

$$
\mathrm{mAIC}=-2 \log \ell(\hat{\beta}, \hat{\theta})+2 \mathrm{k},
$$

where $\mathrm{k}=\mathrm{p}+1+\mathrm{d}$ is the number of unknown parameters and $\mathrm{d}$ is the number of unknown parameters in $\mathbf{D}$.

Besides the marginal perspective, there is also the conditional perspective. In this case we consider the model:

$$
\mathbf{Y} \mid \mathbf{u} \sim \mathrm{N}\left(\mathbf{X} \boldsymbol{\beta}+\mathbf{Z} \mathbf{u}, \sigma^{2} \mathbf{I}_{\mathrm{n}}\right),
$$

Random effects are subject-specific effects on the mean that vary within the population and are estimated subject to a regularization constraint. For such an approach, following Vaida and Blanchard (2005), it can be shown that an 
asymptotically unbiased estimator of the conditional AIC (cAIC) can be introduced as:

$$
\mathrm{cAIC}=\mathrm{n} \log (2 \pi)+\mathrm{n} \log \left(\hat{\sigma}^{2}\right)+\frac{1}{\hat{\sigma}^{2}}(\mathbf{Y}-\mathbf{X} \hat{\boldsymbol{\beta}}+\mathbf{Z} \hat{\mathbf{u}})^{\prime}(\mathbf{Y}-\mathbf{X} \hat{\boldsymbol{\beta}}+\mathbf{Z} \hat{\mathbf{u}})+2(\rho+1),
$$

where

$$
\rho=\operatorname{tr}\left(\left(\begin{array}{cc}
\mathbf{X}^{\prime} \mathbf{X} & \mathbf{X}^{\prime} \mathbf{Z} \\
\mathbf{Z}^{\prime} \mathbf{X} & \mathbf{Z}^{\prime} \mathbf{Z}+\sigma^{2} \mathbf{D}^{-\mathbf{1}}
\end{array}\right)^{-1}\left(\begin{array}{cc}
\mathbf{X}^{\prime} \mathbf{X} & \mathbf{X}^{\prime} \mathbf{Z} \\
\mathbf{Z}^{\prime} \mathbf{X} & \mathbf{Z}^{\prime} \mathbf{Z}
\end{array}\right)\right.
$$

Greven and Kneib (2010) showed that the mAIC is not an asymptotically unbiased estimator of the Akaike information, and favors smaller models without random effects. In turn, for the cAIC, they showed that ignoring estimation uncertainty in the random effects covariance matrix, as is common practice, induces a bias that can lead to the selection of any random effect not predicted to be exactly zero.

The last criterion that we wish to consider is a corrected version of cAIC (ccAIC) proposed by Liang et al. (2008). The correction in the ccAIC concerns the degrees of freedom $\rho$ which are replaced by $\Phi_{0}$ of the form:

$$
\Phi_{0}=\sum_{i=1}^{n} \frac{\partial \hat{Y}_{i}}{\partial Y_{i}}=\operatorname{tr}\left(\frac{\partial \hat{Y}}{Y}\right)
$$

for known $\sigma^{2}$. For unknown $\sigma^{2}$, they proposed to replace $\rho+1$ by:

$$
\Phi_{1}=\frac{\tilde{\sigma}^{2}}{\hat{\sigma}^{2}} \operatorname{tr}\left(\frac{\partial \hat{Y}}{Y}\right)+\tilde{\sigma}^{2}(\hat{Y}-Y)^{\prime} \frac{\partial \hat{\sigma}^{-2}}{\partial \mathrm{Y}}+\frac{1}{2} \widetilde{\sigma}^{4} \operatorname{tr}\left(\frac{\partial^{2} \hat{\sigma}^{-2}}{\partial Y^{\prime} Y^{\prime}}\right)
$$

where $\tilde{\sigma}^{2}$ is an estimate for the true error variance. This method was computationally cumbersome. The further development by Greven and Kneib (2010) avoids the high computational cost and the imprecision of available numerical approximations.

All computations were performed using the $\mathrm{R}$ platform, version 3.2.0 ( $\mathrm{R}$ Core Team, 2015) together with the packages lme4 (Bates et al., 2015), multcomp (Hothorn et al., 2008), and cAIC4 (Saefken et al., 2014). 


\subsection{Materials}

Pork liver pâté with its fat partially substituted (20\%) with flax oil was used as the research material. The cold-pressed flaxseed oil was purchased from Zakład Wytłaczania Oleju i Wyrobu Kitu Krzysztof Gałkowski (Grodzisk Wlkp., Poland).

Flaxseeds (Linum usitatissimum L.) of the Szafir variety (Polish high- $\alpha$ linolenate flax variety; IHAR, Poland) were used in the study. The seeds were ground using a Retch ZM 200 mill (Retch, Germany; 1 mm sieve). Ground seeds were then defatted by double cold extraction with hexane, as described by Waszkowiak and Rudzińska (2014). Flaxseed ethanolic extract was prepared from the defatted meal according to Waszkowiak et al. (2014). All flaxseed additives, i.e. ground seeds (dnz), defatted meal (dno) and ethanolic extract (de), were stored at $4^{\circ} \mathrm{C}$.

Pork liver pâté with $20 \%$ substitution of fat with flax oil was produced according to the formulation: pork meat (class II) $-43 \%$, scalded fine pork fat $33.6 \%$, flax oil $-8.4 \%$, pork liver $-15 \%$. The other ingredients, i.e. broth $30 \%$, salt $-1.6 \%$, pepper $-0.15 \%$, marjoram $-0.05 \%$ and onion $-0.4 \%$ (percentages of meat-fat raw material) were then added during comminution. The meat batter was then divided into six portions and the selected additives were added to each of the portions as shown in Table 1 . The batter was stuffed into heat-shrink casings. The samples were treated at $85^{\circ} \mathrm{C}$ for $50 \mathrm{~min}$, chilled, and then stored at $4^{\circ} \mathrm{C}$.

\subsection{Analytical methods}

During storage, samples were taken periodically (after 1, 5, 8, 11 and 15 days of storage) and the changes in lipid oxidation and fatty acid composition were monitored. The lipid oxidation was assessed by determination of TBARS (2thiobarbituric acid reactive substances) applying the modified distillation method (Pikul et al., 1989; Pikul, 1993). The results were expressed as mg MDA (malondialdehyde) $\mathrm{kg}^{-1}$. The fatty acid composition was estimated according to 
Table 1. Percentages of the supplements applied during the production process

\begin{tabular}{cccc}
\hline \multicolumn{4}{c}{ Additions $\left(\%^{*}\right)$} \\
\hline Sample no. $\begin{array}{c}\text { Ground } \\
\text { flaxseed mealflaxseed meal }\end{array}$ & $\begin{array}{c}\text { Defatted } \\
\text { Flaxseed ethanolic } \\
\text { extract }\end{array}$ \\
\hline 1 & - & - & - \\
2 & - & - & 0.10 \\
3 & 3 & - & - \\
4 & 3 & - & 0.10 \\
5 & - & 1.5 & - \\
6 & - & 1.5 & 0.10 \\
\hline$*$ & percentage of meat batter &
\end{tabular}

AOCS Official Method Ce 1h-05 (Ratnayake et al., 2006). Fatty acid methyl esters were separated using a Hewlett-Packard 5890 II gas chromatograph equipped with a Supelcowax 10 capillary column (30 m x $0.20 \mathrm{~mm} \times 0.20 \mu \mathrm{m}$; Supelco, Bellefonte, PA, USA) and an FID detector. Separated fatty acid methyl esters were identified by comparing their retention data with those of corresponding standards.

\section{Results}

In this model we use the sample number as a random covariate. At the first step we analyzed a full model with all fixed variables considered as fixed covariates: ground seeds (dnz), defatted meal (dno), ethanolic extract (de) and storage time (cp). The variables dnz, dno and de are discrete variables with possible values 0 and 1 , whereas $\mathrm{cp}$ is a continuous variable. In this case we estimated the coefficients for each variable and tested for their significance separately by a simultaneous test procedure as described by Hothorn et al. (2008), implemented in the R package multcomp. Results are presented in Table 2.

The $\mathrm{z}$ statistic is calculated as the estimated value divided by the standard error. 
Table 2. Summary for the model with TBARS factor

\begin{tabular}{|c|c|c|c|c|}
\hline \multicolumn{5}{|c|}{ TBARS $\sim \mathrm{cp}+\mathrm{dnz}+\mathrm{dno}+\mathrm{de}+(1 \mid \mathrm{id})$} \\
\hline & estimate & std error & $\mathrm{Z}$ & adjusted p-value \\
\hline$($ Intercept $)=0$ & 1.561 & 0.165 & 9.456 & $<2 \mathrm{e}-16 * * *$ \\
\hline $\mathrm{cp}=0$ & 0.243 & 0.0304 & 7.999 & $1.33 \mathrm{e}-15 * * *$ \\
\hline $\operatorname{dn} z=1$ & -0.494 & 0.168 & -2.933 & $0.00335 * *$ \\
\hline dno $=1$ & 0.139 & 0.168 & 0.825 & 0.40960 \\
\hline $\mathrm{de}=1$ & -0.138 & 0.138 & -1.002 & 0.31638 \\
\hline
\end{tabular}

Based on the obtained p-values we can conclude that the effects of defatted meal (dno) and ethanolic extract (de) are not significant. The factors with significant effects are storage time (cp) and ground seeds (dnz). The former increases the lipid oxidation, whereas the latter decreases it. Moreover, we would like to verify whether the random effect is relevant. For this purpose we created a linear fixed model without the individual effect, and compared it with the previous one. Table 3 presents the mAIC, cAIC and ccAIC values obtained.

Table 3 shows that the mixed models gave the better fit. Depending on the measure of comparison, the best fit is obtained for the full model in the case of cAIC, whereas ccAIC indicates that the best fit is obtained for the reduced model including storage time and ground seeds.

Table 3. Comparison of fixed and mixed models for TBARS factor

\begin{tabular}{cccc}
\hline Model & mAIC & cAIC & ccAIC \\
\hline TBARS $\sim \mathrm{cp}+\mathrm{dnz}+\mathrm{dno}+\mathrm{de}+(1 \mid \mathrm{id})$ & 18.023 & 18.458 & 10.934 \\
TBARS $\sim \mathrm{cp}+\mathrm{dnz}+(1 \mid$ id $)$ & 15.507 & 19.064 & 9.155 \\
TBARS $\sim \mathrm{cp}+\mathrm{dnz}$ & 18.418 & - & - \\
\hline
\end{tabular}

\section{Discussion}

The mixed model was found to have a useful application in the analysis of the storage of liver pâté with partial substitution of fat with flax oil. Based on mAIC it was shown that mixed-effect models have a better fit than the fixed model. 
However, making the decision based on this criterion for a mixed-effect model may lead to bias, since mAIC assumes a parameter space $\mathrm{R}^{\mathrm{k}}$ and independent and identically distributed observations. The mAIC is thus impaired if the conditions are not fulfilled: it will choose the smaller model more often. On the other hand, Vaida and Blanchard (2005) have shown that the cAIC is asymptotically unbiased for the expected relative Kullback Leibler distance for a given random effects covariance matrix. However, there are some difficulties with the application of cAIC. The problem is that the degrees of freedom in the cAIC are estimated from the same data as the model parameters. The cAIC tends to select too many variables, as could be observed in our example. Liang et al. (2008) propose that the degrees of freedom in cAIC be replaced, resulting in a version called ccAIC. As Greven and Kneib (2010) showed, the ccAIC exhibits satisfactory theoretical properties and is the recommended criterion for a mixed-effect model.

\section{Acknowledgements}

The research was financially supported by the National Science Centre in Poland (Project No. N_N312_242438). The authors are grateful to the anonymous referee for the valuable comments and constructive suggestions.

\section{REFERENCES}

Akaike H. (1974): A new look at the statistical model identification. IEEE Transactions on Automatic Control 19: 716-723.

Bates D., Maechler M., Bolker B., Walker S. (2015): Fitting Linear Mixed-Effects Models Using lme4. Journal of Statistical Software 67(1): 1-48. doi: 10.18637/ jss.v067.i01.

Greven S., Kneib T. (2010): On the Behaviour of Marginal and Conditional AIC in Linear Mixed Models. Biometrika 97(4): 773-789, www.jstor.org/stable/29777136.

Hothorn T., Bretz F., Westfall P. (2008): Simultaneous inference in general parametric models. Biometrical Journal 50(3): 346-363.

Liang H., Wu H., Zou G. (2008): A Note on Conditional AIC for Linear Mixed-Effects Models. Biometrika 95(3): 773-778, doi:10.1093/biomet/asn023.

Pikul J. (1993): Ocena technologiczna surowców i produktów przemysłu drobiarskiego. Wyd. AR. Poznan 104. 
Pikul J., Leszczyński F.A., Kummerow D.E. (1989): Evaluation of three modified TBA methods for measuring lipid oxidation in chicken meat. J. Agric. Food Chem. 37: 1309-1313.

R Core Team (2015): R: A Language and Environment for Statistical Computing. R Foundation for Statistical Computing. Vienna, Austria. url: http://www.Rproject.org/.

Ratnayake N., Hansen S.L., Kennedy M.P. (2006): Evaluation of the CPSil 88 and SP2560 GC columns used in the recently approved AOCS official method Ce 1h-05: Determination of cis-, trans-, saturated, monounsaturated, and polyunsaturated fatty acids in vegetable or non-ruminant animal oils and fats by capillary GLC method. J. Am. Oil Chem. Soc. 83(6): 475-488.

Saefken B., Ruegamer D. with contributions from Greven S., Kneib T. (2014): cAIC4: Conditional Akaike information criterion for lme4. $\mathrm{R}$ package version 0.2 . https://CRAN.R-project.org/package $=$ cAIC4

Vaida F., Blanchard S. (2005): Conditional Akaike information for mixed-effects models. Biometrika 92(2): 351-370.

Waszkowiak K., Rudzinska M. (2014): Effect of flaxseed meals and extracts on lipid stability in a stored meat product. J. Am. Oil Chem. Soc. 9: 979-987.

Waszkowiak K., Szymandera-Buszka K., Hes M. (2014): Effect of ethanolic flax (Linum usitatissimum L.) extracts on lipid oxidation and changes in nutritive value of frozen-stored meat products. Acta Sci. Pol., Technol. Aliment 13(2): 135-144. 